\title{
Cooperative evolution of two different TEs results in lineage-specific novel transcripts in the BLOC1S2 gene
}

Hyeon-Mu Cho ${ }^{1,2+}$, Sang-Je Park' ${ }^{1 \dagger}$, Se-Hee Choe ${ }^{1,2+}$, Ja-Rang Lee ${ }^{3}$, Sun-Uk Kim²,4, Yeung-Bae Jin ${ }^{1}$, Ji-Su Kim²,3, Sang-Rae Lee ${ }^{1,2}$, Young-Hyun Kim ${ }^{1,2^{*}}$ and Jae-Won Huh ${ }^{1,2^{*}}$

\begin{abstract}
Background: The BLOC1S2 gene encodes the multifunctional protein BLOS2, a shared subunit of two lysosomal trafficking complexes: i) biogenesis of lysosome-related organelles complex-1 and i) BLOC-1-related complex. In our previous study, we identified an intriguing unreported transcript of the BLOC1S2 gene that has a novel exon derived from two transposable elements (TEs), MIR and AluSp. To investigate the evolutionary footprint and molecular mechanism of action of this transcript, we performed PCR and RT-PCR experiments and sequencing analyses using genomic DNA and RNA samples from humans and various non-human primates.
\end{abstract}

Results: The results showed that the MIR element had integrated into the genome of our common ancestor, specifically in the BLOC1S2 gene region, before the radiation of all primate lineages and that the AluSp element had integrated into the genome of our common ancestor, fortunately in the middle of the MIR sequences, after the divergence of Old World monkeys and New World monkeys. The combined MIR and AluSp sequences provide a 3' splice site (AG) and 5' splice site (GT), respectively, and generate the Old World monkey-specific transcripts. Moreover, branch point sequences for the intron removal process are provided by the MIR and AluSp combination.

Conclusions: We show for the first time that sequential integration into the same location and sequence divergence events of two different TEs generated lineage-specific transcripts through sequence collaboration during primate evolution.

Keywords: Primate, Transposable element, Sequential integration, Alternative splicing, Branch point

\section{Background}

BLOC1S2, located on human chromosome 10q24.31, encodes the multifunctional protein BLOS2 that is a shared subunit of two lysosomal trafficking complexes. The first is biogenesis of lysosome- related organelles complex 1 (BLOC-1), which functions in the generation of specialized organelles in the endosomal-lysosomal system, such as platelet dense granules or melanosomes. The other is BLOC-1-related complex (BORC), which has been reported to regulate the positioning of lysosomes $[1,2]$. In

\footnotetext{
* Correspondence: kyh@kribb.re.kr; huhjw@kribb.re.kr

${ }^{\dagger}$ Hyeon-Mu Cho, Sang-Je Park and Se-Hee Choe contributed equally to this work.

${ }^{1}$ National Primate Research Center, Korea Research Institute of Bioscience and Biotechnology (KRIBB), Cheongju 28116, Korea

Full list of author information is available at the end of the article
}

addition, BLOS2 is involved in several cellular processes independent of BLOC-1 and BORC [1], namely i) the inhibition of the transcriptional suppression activity of BRD7, which is a tumor suppressor candidate [3]; ii) negative regulation of Notch signaling, which is a highly conserved cell-to-cell signaling pathway [1]; and iii) the regulation of apoptotic cell death as the result of interacting with HIPPI (HIP-1 protein interactor) $[1,2]$. In addition, BLOS2 is widely expressed both in normal tissue and malignant tumors with a tendency towards lower expression levels in certain tumor subtypes [2].

Alternative splicing (AS) is a post-transcriptional process that contributes significantly to eukaryotic gene diversity by creating multiple isoforms without increasing the genome size $[4,5]$. AS is performed by a

(c) The Author(s). 2019 Open Access This article is distributed under the terms of the Creative Commons Attribution 4.0 International License (http://creativecommons.org/licenses/by/4.0/), which permits unrestricted use, distribution, and reproduction in any medium, provided you give appropriate credit to the original author(s) and the source, provide a link to the Creative Commons license, and indicate if changes were made. The Creative Commons Public Domain Dedication waiver (http://creativecommons.org/publicdomain/zero/1.0/) applies to the data made available in this article, unless otherwise stated. 
spliceosome coupled with splicing regulators that bind to cis-acting elements of the target pre-mRNA [6]. The AS mechanism is classified into several types: exon skipping (cassette exons), alternative $5^{\prime}$-splice site (5'-SS), alternative 3 '-splice site ( $3^{\prime}$ - SS), intron retention, mutually exclusive exons, alternative promoter, and alternative polyadenylation $[4,7-9]$. In the evolutional view, an alternatively spliced exon can be generated by three different molecular mechanisms: exon shuffling, transition of a constitutive exon to an alternative exon, and exonization of intronic sequences [10-12].

Particularly, exonization exploits repetitive mobile elements called transposable elements (TEs) for the event itself. Of the 3 billion base pairs in the human genome, about $45 \%$ are made up of TEs, and about $4 \%$ of human genes contain TE motifs in their coding region, which suggests an exonization event $[8,12]$. TEs are divided into several types, such as DNA transposons (3\%), long interspersed nuclear elements (LINEs, 21\%), short interspersed nuclear elements (SINEs, 13\%), and human endogenous retroviruses (HERVs, 8\%) [13-15]. Specifically, Alu and MIR, which belong to the SINE family, are known to contribute to alternative splicing [16]. The Alu element, the most abundant TE in the human genome, is primate specific and composed of two similar monomers, the left and right arms, and these arms in the antisense orientation provide potential $5^{\prime}$ - and $3^{\prime}$-splicing sites that can be recognized by the spliceosome $[17,18]$. Moreover, a previous study has shown that Alu creates $\sim 5 \%$ of the alternatively spliced exons in the human genome; thus, it is considered crucial for AS events [19]. MIR is the second most common SINE in primates, representing $\sim 2.5 \%$ of the human genome, and is interspersed within the mammalian genome $[20,21]$. It also provides possible splicing sites for the inserted loci in the antisense orientation [16]. Therefore, TE- derived AS events are crucial sources for multi-transcript creation.

The aims of the present study were the identification and molecular characterization of MIR_AluSp-derived exonization events in the BLOC1S2 gene from the evolutionary point of view. Accordingly, we analyzed the approximate integration time of both the MIR and Alu elements into the BLOC1S2 gene using sequencing methods and conducted comparative analyses of the expression patterns of the exonized target sequence in various tissues from rhesus monkey (Macaca mulatta), crab-eating monkey (Macaca fascicularis), and humans (Homo sapiens) to closely examine their interspecies differences. Consequently, this study determined the mechanism by which these two different TEs combined and were involved in the lineage-specific AS event during primate evolution.

\section{Results}

Comparative analysis of the structure of the BLOC1S2 gene in humans and non-human primates

In a previous study, a large-scale transcriptome sequencing analysis of the genome of the crab-eating monkey enabled us to detect a new partial exon, which is located on the 4th intron of the BLOC1S2 gene, overlapping sequences of two TEs, AluSp, and MIR (Fig. 1) [22]. No transcript variants including this partial exon exist in the UCSC Genome Browser database for the crab-eating monkey, rhesus monkey, and human. Prior to identification and experimental validation of this partial exon, we investigated the BLOC1S2 mRNA sequence of the crabeating monkey and analyzed its structure comparatively with that of the rhesus monkey and human mRNA sequences. The crab-eating monkey BLOC1S2 gene transcript (NM_001287735.1) is composed of five exons and transcribes into a mRNA of $1208 \mathrm{bp}$ containing a 19-bp 5 '-untranslated region (UTR), a 429-bp coding sequence (CDS), and a 760-bp 3'-UTR. The same conserved five exons also constitute the BLOC1S2 gene transcripts of both the rhesus monkey (NM_001266915.1) and human (NM_001282439.1), which transcribe into mRNAs of $1158 \mathrm{bp}$ and $2663 \mathrm{bp}$, respectively (Additional file 1: Figure S1). The notable difference among these three transcripts is the relatively shorter length of the UTRs in the rhesus monkey and crab-eating monkey compared to that of the human UTR. Further, the coding sequence identity was calculated using NCBI Blast, revealing that the $B L O C 1 S 2$ gene transcripts of the crab-eating monkey and rhesus monkey were $99 \%$ identical and that both were $96 \%$ identical to the human BLOC1S2 gene transcript.

\section{Evolutionary analysis of the MIR and AluSp integration event in the BLOC1S2 gene}

To investigate the integration time of the target MIR and AluSp element, we experimentally analyzed genomic DNA samples from 10 primates, including hominoids (human, chimpanzee, and gibbon), Old World monkeys (rhesus monkey, crab-eating monkey, African green monkey, and colobus monkey), New World monkeys (marmoset and squirrel monkey), and prosimian (ringtailed lemur). We performed genomic PCR amplification of these samples (Fig. 2a and b) and obtained their sequences using gene-cloning methods. The alignment of these sequences revealed that the MIR is located in an antisense-orientation on the 4th intron of the BLOC1S2 gene in all tested primates and that the antisenseoriented AluSp is located in the same region, but only in the hominoids and Old World monkeys (Additional file 1: Figure S2). Notably, AluSp exists inside the MIR sequence. Unlike the hominoids and Old World monkeys, the New World monkeys and prosimians do not contain 


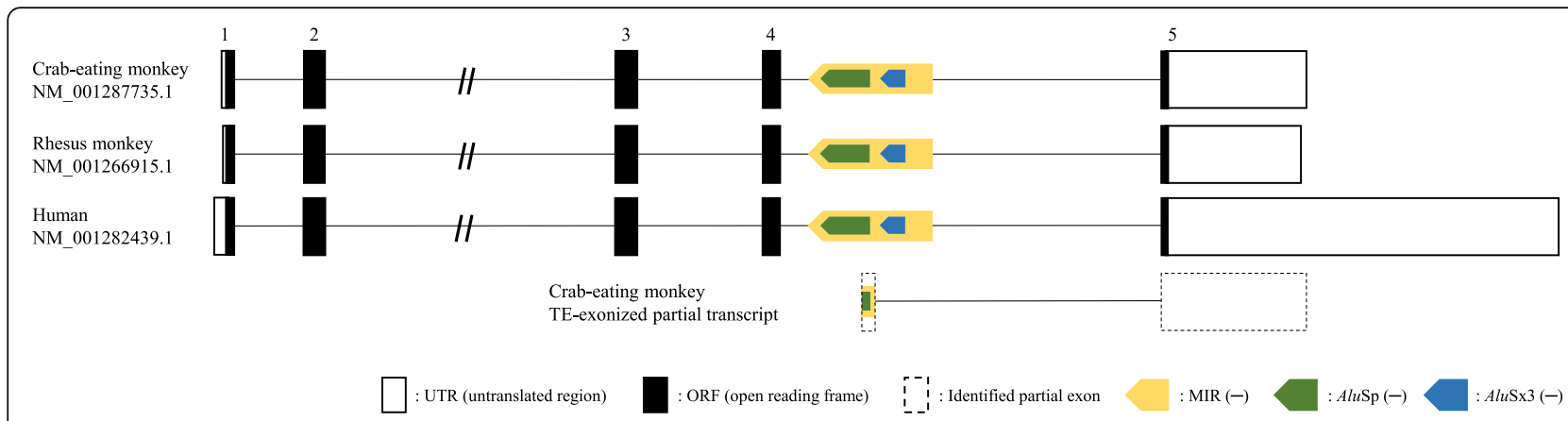

Fig. 1 Structural analysis of BLOCIS2 of the crab-eating monkey, rhesus monkey, and human. The MIR and AluSp are located in the antisense orientation on the 4th intron of BLOC1S2. Open, closed, and dashed line boxes represent the untranslated region of the exon, coding sequence, and identified partial exon, respectively. Yellow, green, and blue arrow boxes represent MIR, AluSp, and AluSx3, respectively. This figure is a structural illustration and is not drawn to scale

a $260 \mathrm{bp}$ AluSp element, but possess a different Alus at different loci within the target PCR products, which results in similar sizes of the 10 main PCR amplicons (Fig. 2b and Additional file 1: Figure S3). Taken together, these evolutionary analyses indicate that the MIR element was integrated into the genome of simians and prosimians prior to the divergence of their common ancestor, approximately more than 63 million years (myrs) ago. In addition, the AluSp element was integrated into the genome of hominoids and Old World monkeys after they split off from their common ancestors with New World monkeys somewhere between 25 and 40 myrs ago (Fig. 2c).

\section{Experimental validation of MIR_Alu-derived exonization events in the BLOC1S2 gene}

Both ends of the newly detected partial exon were not correctly identified, making it necessary to perform RT-

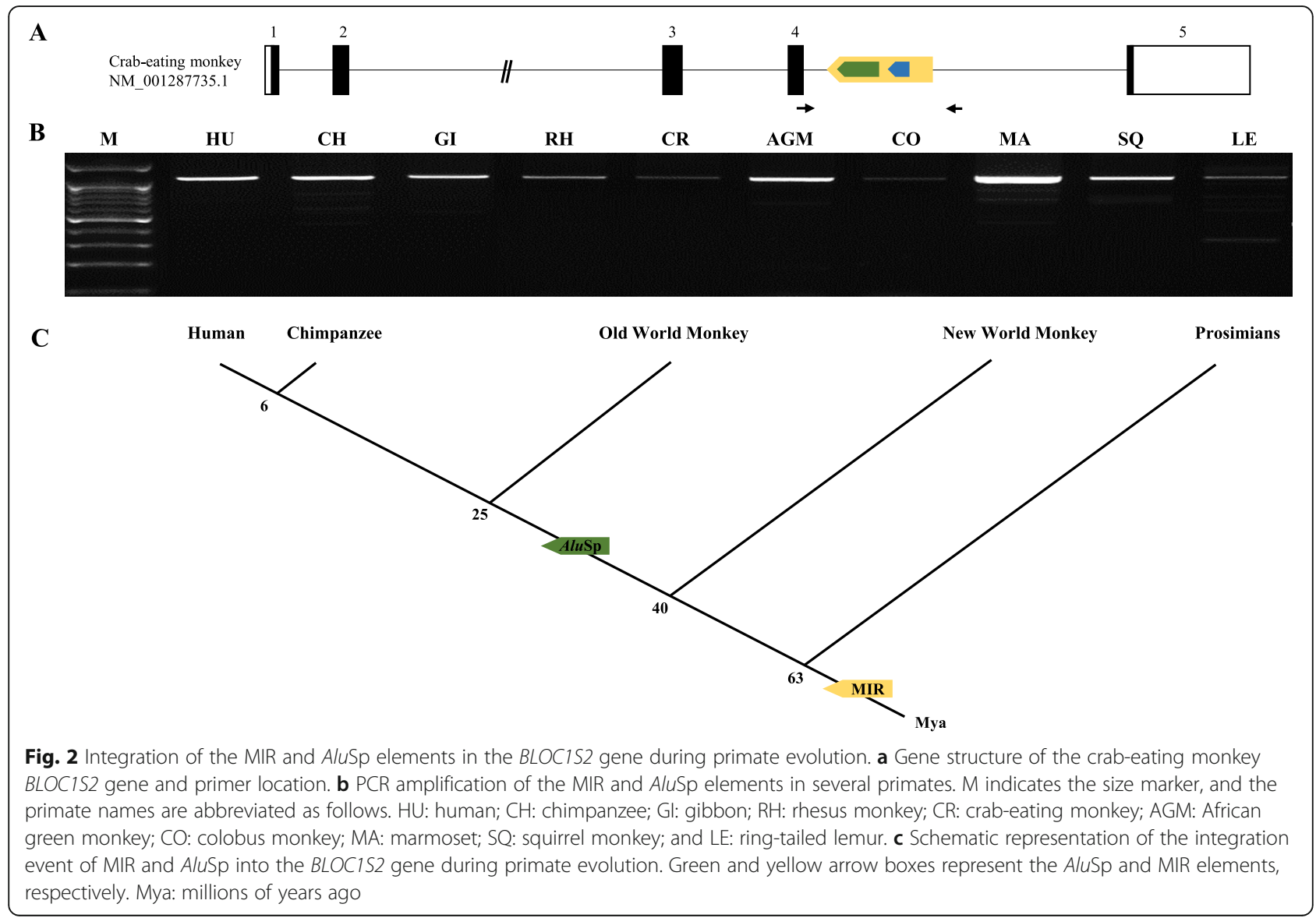


PCR experiments to validate both ends. Tissue cDNA samples from the crab-eating monkey were used, and two primer pairs were designed: i) Validation Primer 1 was used to find the $5^{\prime}$-end of the partial exon and 2) Validation Primer 2 was used to check the 3 '-end of this partial exon (Additional file 1: Table S1). As a result, we clarified the sequence of the full length of the partial exon, finding that the $5^{\prime}$-end is spliced at the 3'-SS (AG) derived from the AluSp sequence on the 4th intron of the gene and that the $3^{\prime}$-end is spliced at the 5'-SS (GT) derived from the MIR sequence on the same intron (Fig. 3 and Additional file 1: Figure S2). We named this exon V1. In addition, to determine if the exon V1 exists in other species of primates, several more rounds of RT-PCR and sequencing were carried out with the available human, chimpanzee, gibbon, rhesus monkey, and African green monkey tissues (Additional file 1: Figure S4). The V1 transcript was detected in the rhesus monkey and African green monkey, but not in human, chimpanzee, and gibbon (Additional file 1: Figure S4A). For human, chimpanzee, and gibbon, numerous nonspecific amplicons were detected (Additional file 1: Figure S4B); therefore, several of these amplicons of acceptable size were sequenced, but they were unrelated to V1. Furthermore, we found that a $506 \mathrm{bp}$ amplicon that was exon V1 but with a different 3 '-SS (AG) loci provided by the same AluSp in the cerebrum of the African green monkey (Additional file 1: Figure S4A and Fig. 3). We named it V2, and a new primer pair, Primer 3 (Additional file 1: Table S1), was applied to check the ends of V2. We confirmed that V2 had a different 3'-SS, and its expression was experimentally validated in the African green monkey, crab-eating monkey, and rhesus monkey, but not in human, chimpanzee, and gibbon (Additional file 1: Figure S5).

\section{Expression patterns of the MIR_Alu-derived exon in various primates}

To clarify the expression patterns of the original, V1, and V2 transcripts, we performed RT - PCR amplification of this region using various tissue cDNA samples from the crab-eating monkey, rhesus monkey, and human. The results revealed a ubiquitous expression pattern of the original transcript in all tissue samples from the crab-eating monkey, rhesus monkey, and human; however, the expression level was relatively low in the pancreas of the crab-eating monkey, the kidney, and liver of the rhesus monkey, and the human liver and colon (Fig. 4). V1 was expressed in all the tissue samples from the crab-eating monkey, with a low level of expression in the pancreas. However, very low or no V1 expression was found in the tissue samples from the rhesus monkey. In case of human tissues, V1 was not detected in any of the tissue samples analyzed (Fig. 4c). Thus, we designed and applied a new primer called Primer 5 (Additional file 1: Table S1) to detect V1 more precisely in case there were very low expression levels in human tissues, but no variants related to V1 were detected (data not shown). RT-PCR was used to detect V2 with the same tissue cDNA samples, and the results revealed that V2 is partly expressed in tissue samples from the crabeating monkey and rhesus monkey (Fig. 4 and Additional file 1: Figure S6). Specifically, no V2 was expressed in the kidney, stomach, spleen, small intestine, pancreas, heart, and spinal cord of the crab-eating monkey and in the kidney, liver, stomach, and pancreas of

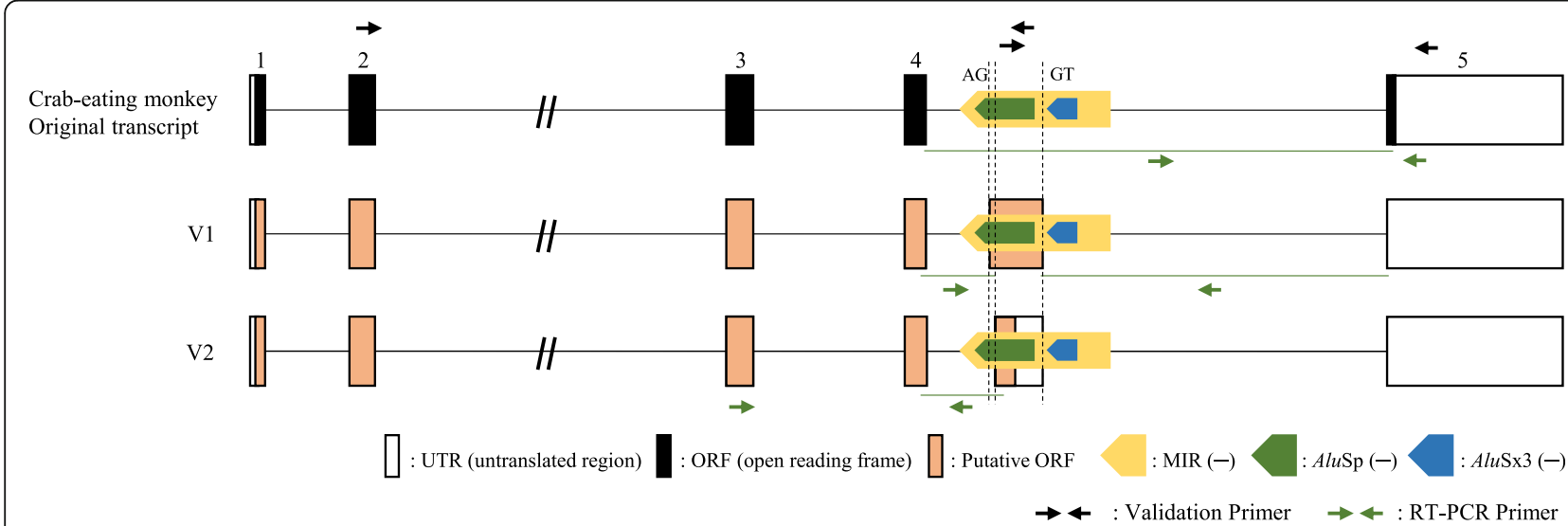

Fig. 3 Structural analysis of BLOC1S2 gene transcripts in the crab-eating monkey, rhesus monkey, and African green monkey through RT-PCR experiments and sequencing of the products. The two transcript variants (V1 and V2) attributed to MIR_AluSp-derived exonization events were identified in all species. Each arrow box with a different color indicates a different SINE element. Open and closed boxes represent the untranslated region of the exon and coding sequence, respectively. Yellow, green, and blue arrow boxes represent MIR, AluSp, and AluSx3, respectively. Black and green small arrows represent the validation primer and RT-PCR primers, respectively. This figure is a structural illustration and is not drawn to scale 


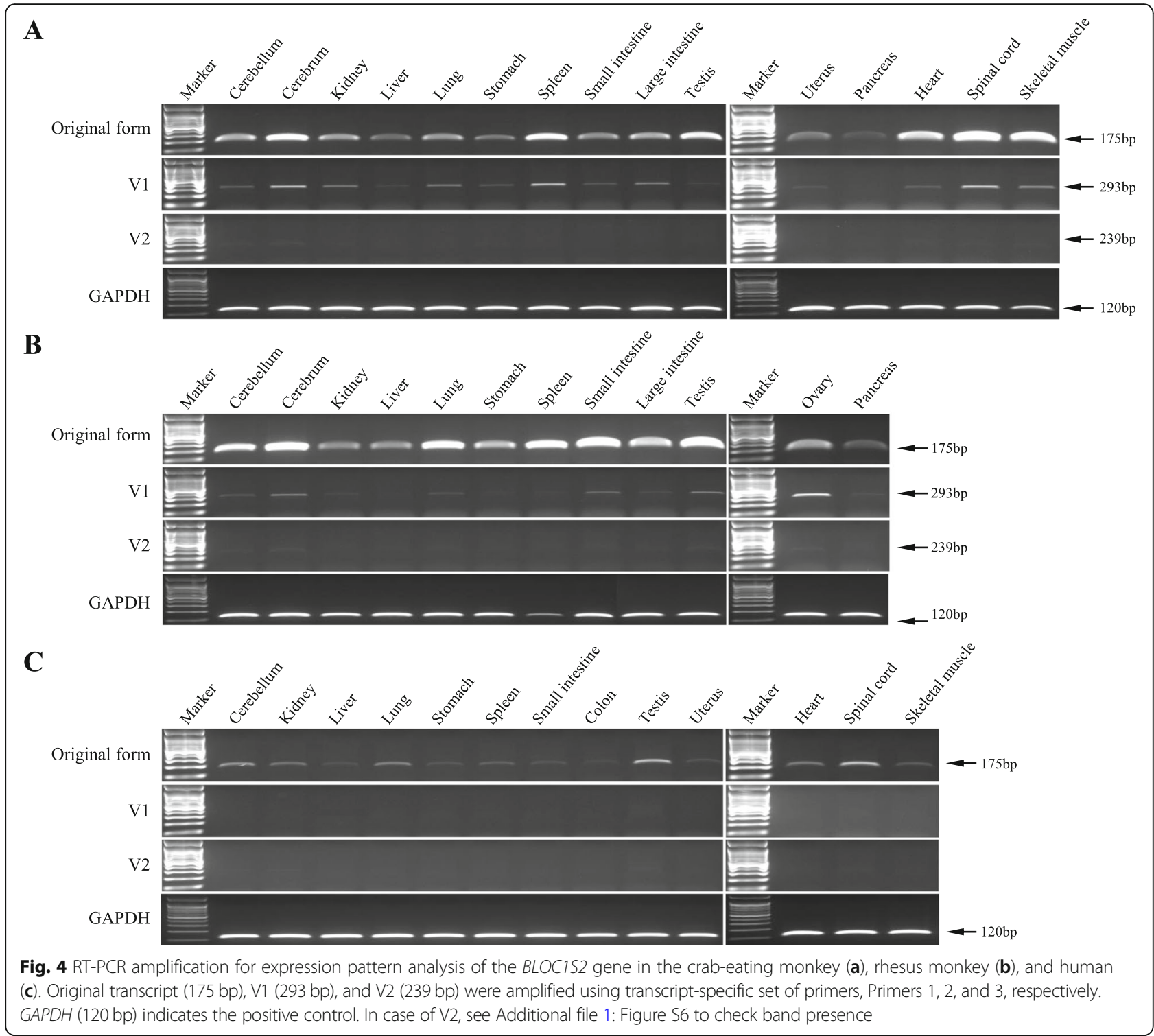

the rhesus monkey. On the other hand, V2 was expressed in the cerebellum, cerebrum, lung, large intestine, and testis of the two species (Additional file 1: Figure S6). In human tissues, we were able to detect six faint amplicons that were of a similar size (277 bp) to the target band (239 bp) (Additional file 1: Figure S6), but we confirmed by cloning that these bands were not the target V2.

\section{Peptide sequence analysis of BLOC1S2}

The translated sequence analysis using the ORF finder program revealed that the original, V1, and V2 transcripts encode 142, 222, and 160 amino acids, respectively (Additional file 1: Figure S7). Additionally, domain search analysis using the Pfam database showed that a conserved domain named BLOC1_2 exists on the original transcript located from the 42nd amino acid to the 137th amino acid. For V1 and V2, however, the last five amino acids of this domain are replaced with a longer and different amino acid sequence (Additional file 1: Figure S7). A close look at these amino acid sequences being compared with the equivalent transcript sequences uncovered that these different amino acid sequences are derived from the inserted MIR_AluSp, providing premature stop codon (Fig. 5).

\section{Discussion}

Non-human primates are very valuable animal models for biomedical research because of their biological, behavioral, physiological, and genetic similarities with humans [23, 24]. Among these primates, crab-eating monkeys, rhesus monkeys, and African green 


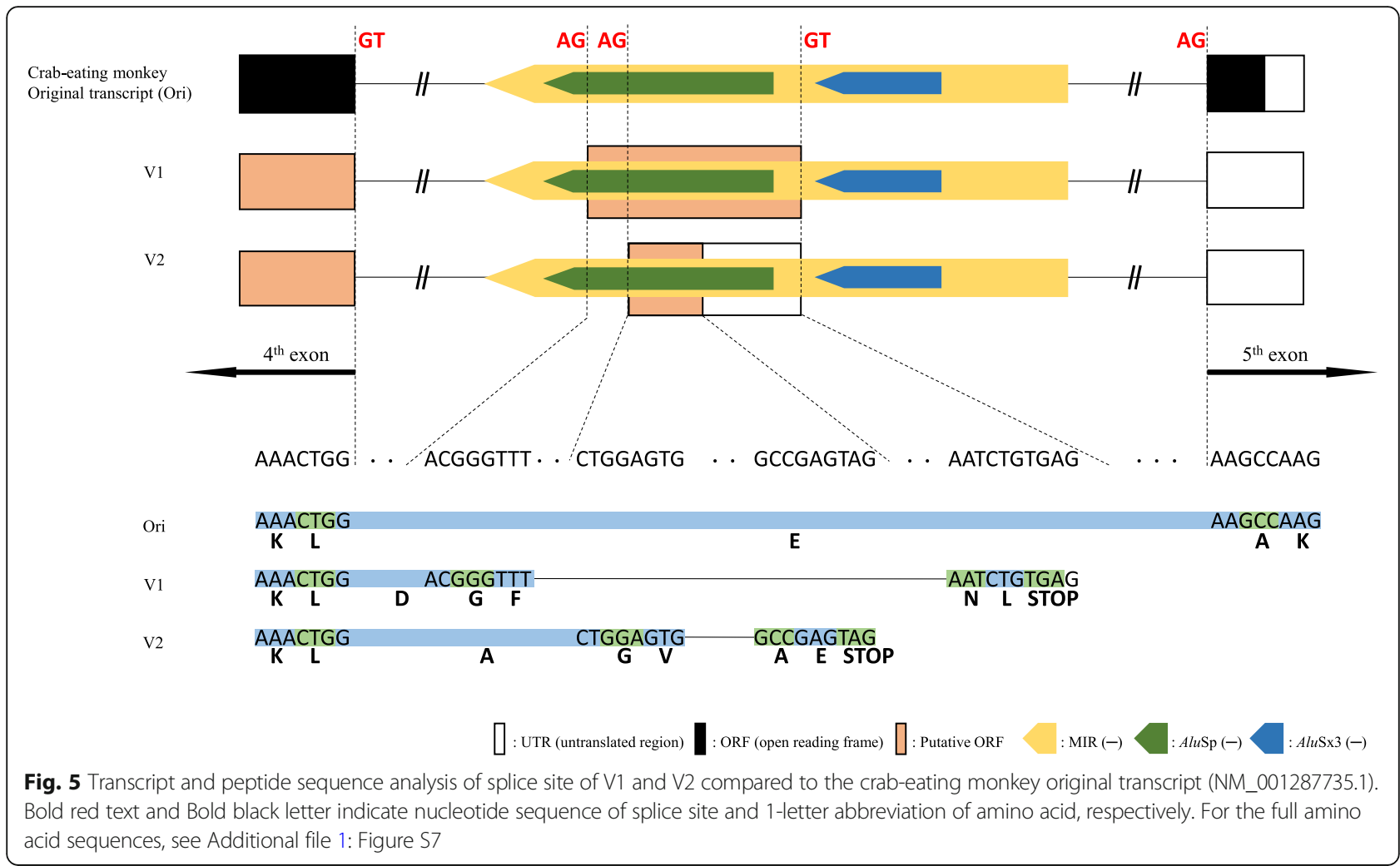

monkeys are the most representative and frequently used experimental animals in biomedical research [23]. These monkeys are Old World monkeys and diverged from the common ancestor of Old World monkeys and humans approximately 25-32 myrs ago [24].

TEs have moved around the genome over the past hundreds of million years and shaped the evolution of the human and non-human primate genomes $[25,26]$. Previous studies have indicated that TE insertions are associated with more than 100 diseases to date because of their association with increasing genomic instability [27, 28]. Several disease-causing mechanisms have been identified such as insertional mutagenesis and aberrant splicing $[25,28]$. On the other hand, TEs can be advantageous elements that increase genomic diversity, and have a tremendous impact on evolution at the DNA and RNA levels by transduction-mediated gene formation, gene retrotransposition, alternative splicing, alternative polyadenylation, and the actions of alternative promoters, enhancers, and silencer elements $[11,18,25]$. In the present study, for the first time, we identified and validated that MIR_AluSp combined sequences induced lineage-specific alternative splicing events and played an important role in diversifying BLOC1S2 gene transcripts, especially in the crab-eating monkey, rhesus monkey, and African green monkey.

\section{Insertion mechanism of MIR and AluSp in BLOC1S2}

The target exonization that was the subject of this study derives from combined sequences. Three SINE elements, two Alus and one MIR, are involved in this combination, and two of these elements, AluSp and MIR, are associated with the target exonization event. To investigate the collaborative mechanism involving these two TE insertion events, genomic DNA cloning and sequence analyses were conducted. The most plausible scenario for the integration event of the TEs based on thorough sequence analyses is as follows. The target MIR element was integrated into the 4th intron of the BLOC1S2 gene in all analyzed primates prior to mammalian radiation [13], and then, AluSp was inserted into the endonuclease target motif located on the MIR of the catarrhine lineage before the divergence of hominoids and Old World monkeys, creating the combined MIR_AluSp sequence $[20,21]$.

The multiple alignment (Additional file 1: Figure S2) enabled us to analyze the insertion mechanism of the two elements. The main insertion mechanism that the SINE family usually adopts is called the target-primed reverse transcription (TPRT) $[25,29,30]$. This mechanism of insertion by $A l u$ normally leaves the two following hallmarks in the genome: i) a target site duplication (TSD) and ii) a variable length poly-A tail [31]. The sequence analysis revealed that the target $A l u S p$ was inserted into the genome by the TPRT mechanism 
because it exhibited these two characteristics (Additional file 1: Figure S2). MIR has also been reported to have flanking repeats such as a TSD and an A/T-rich 3 '-end, such as a poly-A-tail [20]. In the case of the A/T-rich 3 '-end, it is still discernible. On the other hand, we were able to find three matched nucleotides directly in front and behind the MIR sequence that look like flanking repeats (Additional file 1: Figure S2). This could be the TSD according to a previous study that defined the length of a TSD as being between 2 and $20 \mathrm{bp}$ [25]. Nevertheless, we cannot be sure that this is the TSD generated by MIR insertion because it seems to have become unrecognizable due to its $25-35 \%$ divergence from the consensus sequence [20].

\section{Alternative splicing events by combined TEs}

Previous studies have shown that $\mathrm{Alu}$ and MIR elements were preferentially inserted in the antisense orientation and sense orientation, respectively, relative to the gene orientation. Moreover, 60 and $85 \%$ of the exonized sequences from MIR and Alu, respectively, are in the antisense orientation [16]. The genomic DNA sequence analysis performed in this study showed that the target MIR and AluSp elements were both in the antisense orientation and combined together and that two 3'-SS and one $5^{\prime}$-SS were derived from these elements, respectively (Figs. 3 and 5). Therefore, the combined MIR AluSp elements in antisense-orientation can lead to AS events and exonization by providing potential splicing donor and acceptor sites in the intragenic regions. Thus, we conducted additional computational analyses to clarify the distribution of the combined MIR_AluSp elements at different loci in the genome of the crab-eating monkey, rhesus monkey, and human (Additional file 1: Table S2).

In the case of the MIR_AluSp combination of the crab-eating monkey, 209 cases were detected, and 60\% (125cases) were in the same orientation. Additionally, we tried to calculate the number of MIR_AluSp combinations that were intragenic although the gene data files we used only contain NM_ and NR_genes that have been recently updated. As a result, 4 out of 125 cases, including the analyzed MIR_AluSp combination in the $B L O C 1 S 2$ gene, were both intragenic and intronic. The computing analyses of the rhesus monkey and human showed similar ratios, but different counts, because of the different number of registered genes for different species (Additional file 1: Table S2). This simple analysis revealed that combined MIR_AluSp sequences are distributed inside the genes in the crab-eating monkey, rhesus monkey, and human genomes. Further experiments to determine the number of transcripts created by MIR AluSp sequences will reveal how much influence this specific combined sequence has on the genome. In addition, broad studies on various types of combinations coupled with their exonization may allow the identification of more TE-combined sequences that contribute to AS events and aid in the research on the preference of splice sites.

\section{Molecular mechanism of the MIR_AluSp sequence resulting in alternative splicing}

Splicing is the process by which introns are removed from pre-mRNA transcripts, and three essential cisacting elements, i) a 5'-SS, ii) $3^{\prime}$-SS, and iii) branch point (BP) are required for this intron removal process [32]. The process accompanies two transesterification reactions: the first reaction is involved in the attack of the $\mathrm{BP}$ adenosine on the $5^{\prime}$ splice donor to cut the intron from upstream exon, and the second reaction is involved in the attack of this detached end on the 3 ' splice acceptor, releasing the intron in the form of a lariat [33]. Therefore, the BP recognized by the ribonucleoprotein U2 snRNA plays a crucial role in the initiation of splicing [34].

To analyze the 3 '-SS of the newly identified target exon, we focused on the removal process of the upstream 4th intron. Computational analysis was performed to predict possible BP positions for this intron. For the V1 transcript, 5'-GCTTTAC-3' (V1_BP-A) and 5' - GTATTATGT-3' (V1_BP-B) were detected as the $\mathrm{BP}$ sequence by two different BP prediction tools, BPP and svm-BPfinder, respectively, and the last adenine of each BP sequence is expected to be the BP (Fig. 6). In the case of the V2 transcript, 5'-TTTTGAG-3' (V2_BPA) and 5'-GTTTCATTC-3' (V2_BP-B) were identified as possible $\mathrm{BP}$ sequences, and the adenine of each $\mathrm{BP}$ sequence is considered as the BP (Fig. 6). A previous study reported that the human BP is usually found 21-25 nucleotides (nts) upstream of the 3'-SS or near this region [34-37]. The predicted BP position in this study was also detected near this region (Fig. 6). Interestingly, the upstream BP positions of the target exon in the hominoids and Old World monkeys are located in the same region, although the target exon is only expressed in the Old World monkeys. This may be because the predicted BP sequence of hominoids is not recognized by the U2 snRNP, inhibiting the creation of a downstream target exon.

A previous study revealed that BP selection is highly associated with the distance to the $3^{\prime}$-SS and BP binding stability [36]. First, a BP located farther from the 3'-SS than at the canonical BP position is less likely to be selected, resulting in the skipping of the downstream exon. Corvelo et al. showed that approximately $43 \%$ of the exons in which the BP was located more than $100 \mathrm{nts}$ upstream of the 3'-SS were skipped, while only $28.6 \%$ of exons in which the BP was located less than 50 nts were 


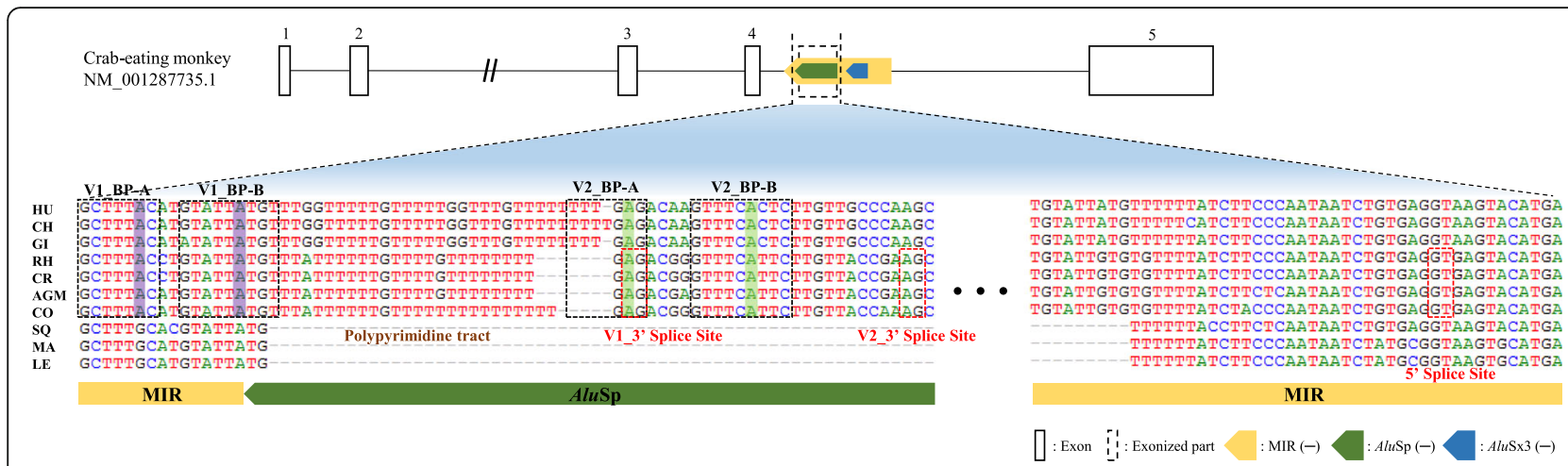

Fig. 6 Branch point (BP) analysis of MIR_AluSp-derived V1 and V2 transcripts using the BPP and svm-BPfinder programs. The purple box is the predicted BP of the V1 transcript, and the green box is the predicted BP of the V2 transcript. The red dashed box represents $3^{\prime}$ splice sites and 5' splice sites. Open and closed boxes represent the untranslated region of the exon and coding sequence, respectively. Yellow, green, and blue arrow boxes represent MIR, AluSp, and AluSx3, respectively. This figure is a structural illustration and is not drawn to scale

skipped [36]. Second, the binding stability of the BP sequence to the binding sequence of the U2 snRNA could be another reason [36]. Recently, the binding energy of this base pairing was calculated with a computing program, which represents binding stability [36]. For example, a $\mathrm{C}$ to $\mathrm{T}$ mutation near the $\mathrm{BP}$ position of the Fech1 gene intron that decreased the U2 binding energy was reported as the cause of the creation of a new competing $\mathrm{BP}$ [38]. Likewise, in the case of the V2 transcript in this study, the BP binding energy in hominoids may be less than that in Old World monkeys. This is because the one nucleotide difference in the V2 BP-B between the hominoids $(\mathrm{C})$ and old World monkeys $(\mathrm{T})$ could decrease its stability, hindering the predicted BP from being selected. In the case of the V1 transcript, the binding stability of a different protein, U2AF (U2 auxiliary factor), may be associated with this selection of the predicted BP. U2AF65, a $65-\mathrm{kDa}$ subunit of U2AF, usually binds to the polypyrimidine tract (Py tract) right behind the $\mathrm{BP}$ sequence and is involved in the recruitment of U2 snRNP [39, 40]. In addition, a previous study revealed that the binding affinity of U2AF65 differs depending on the sequence of the Py tract [41]. Therefore, the different Py tract sequences between hominoids and Old World monkeys in this study could lower the U2AF65 binding energy, thereby interrupting the selection of the BP.

\section{Diverse peptide sequence derived from exonized MIR_AluSp}

Our peptide sequence analysis of the target transcript using ORF finder program and Pfam database identified 2 more possible peptide sequences different from the one that is originated from the reference transcript (NM_001287735.1). It turns out, from the analysis, that these differences are generated by MIR_AluSp exonization and especially centered on C-terminal of these peptide sequences (Fig. 5 and Additional file 1: Figure S7). Previous studies have indicated that different $C$ - terminal lengths of alternatively spliced isoform tend to result in different protein functions [42]. In addition, many predicted alternative transcripts might not be translated into proteins [43]. Thus, further studies are needed to validate whether the two BLOC1S2 transcript variants are functional. Although a functional analysis was not performed in the present study, the specific integration and combination of the MIR and AluSp elements was shown to lead to lineage-specific AS events in the Old World monkeys. Therefore, in this study, we revealed a novel mechanism for exonization events involving collaboration of two different TEs, specifically in a lineagespecific manner.

\section{Conclusion}

Overall, the combined sequence of the two MIR and AluSp elements in antisense orientation in the BLOC1S2 gene favors Old World monkey-specific alternative splicing. In this molecular landscape, the MIR of the Old World monkey is evolutionally designed to provide a premature BP sequence. This BP sequence is also conducive to the determination of the $3^{\prime}$-SS on the AluSp sequence, contributing to the new exon generation inside the 4th intron. The subsequent intron removal process that starts at the $5^{\prime}$-SS on the MIR ends this newly detected target exon. Therefore, the merging of the two MIR and AluSp sequences in antisense orientation produced novel Old World monkey-specific transcript variants by adding a new exon to the BLOC1S2 gene during primate evolution.

\section{Methods}

\section{Total RNA and genomic DNA samples}

Total RNA from human tissue samples (Homo sapiens; whole brain, kidney, liver, lung, stomach, spleen, small 
intestine, colon, testis, uterus, heart, spinal cord, and skeletal muscle) and rhesus monkey tissue samples (Macaca mulatta; cerebellum, cerebrum, kidney, liver, lung, stomach, spleen, small intestine, large intestine, testis, ovary, and pancreas) were purchased from Clontech Laboratories, Inc. Tissues from an adult female crab-eating monkey (Macaca fascicularis; cerebellum, cerebrum, kidney, liver, lung, stomach, spleen, small intestine, large intestine, testis, uterus, pancreas, heart, spinal cord, and skeletal muscle) that originated from Vietnam was imported from China under a Convention on International Trade in Endangered Species of Wild Fauna and Flora (CITES) permit. The crab-eating monkey was provided by the National Primate Research Center (NPRC) of the Republic of Korea. We used a standard protocol to isolate genomic DNA from heparinized blood samples from the following species: (1) hominoids: $\mathrm{HU}$, humans (Homo sapiens); $\mathrm{CH}$, chimpanzees (Pan troglodytes); and GI, gibbon (Symphalangus syndactylus); (2) Old World monkeys: RH, rhesus monkeys (Macaca mulatta), CR, crab-eating monkeys (Macaca fascicularis), AGM, African green monkeys (Chlorocebus aethiops), and CO, colobus monkeys (Procolobus badius); (3) New World monkeys: MAR, marmosets (Callithrix jacchus) and SQ, squirrel monkeys (Saimiri sciureus); and (4) prosimians: LE, ring-tailed lemurs (Lemur catta).

\section{RT-PCR and PCR amplification}

BLOC1S2 transcripts were analyzed by RT-PCR amplification using the GoScript Reverse Transcriptase (RT) System (Promega) with an annealing temperature of $42{ }^{\circ} \mathrm{C}$. We performed PCR amplification of pure mRNA samples without reverse transcription to demonstrate that the mRNA samples did not contain genomic DNA (data not shown). The expression levels of the BLOC1S2 gene of humans, rhesus monkeys, and crab-eating monkeys were tested by RT-PCR experiments using specific primer pairs (Additional file 1: Table S1). RT-PCR experiments were carried out for 30 cycles of $94^{\circ} \mathrm{C}$ for 30 s, $59^{\circ} \mathrm{C}$ for $30 \mathrm{~s}$, and $72{ }^{\circ} \mathrm{C}$ for $30 \mathrm{~s}$. We additionally performed 35 cycles of RT-PCR experiments for V2 transcript due to its dimly visible bands with 30 cycles. Genomic DNA from the different primates was divided into two groups and separately amplified with two different primer pairs, one for human, chimpanzee, and gibbon and another for rhesus monkey, crab-eating monkey, African green monkey, marmoset, squirrel monkey, and ring-tailed lemur. The genomic PCR conditions for the former group were 35 cycles of $94{ }^{\circ} \mathrm{C}$ for 30 s, $58^{\circ} \mathrm{C}$ for $30 \mathrm{~s}$, and $72{ }^{\circ} \mathrm{C}$ for $70 \mathrm{~s}$, and those for the latter group were $34 \mathrm{cycles}$ of $94^{\circ} \mathrm{C}$ for $30 \mathrm{~s}, 58^{\circ} \mathrm{C}$ and $62{ }^{\circ} \mathrm{C}$ for $30 \mathrm{~s}$, and $72{ }^{\circ} \mathrm{C}$ for $60 \mathrm{~s}$.

\section{Molecular cloning and sequencing procedures}

PCR products were separated on a $1.2 \%$ agarose gel, purified with the Gel SV Extraction kit (GeneAll), and cloned into the TA cloning vector (RBC Bioscience). The cloned DNA was isolated using the Hybrid- $\mathrm{Q}^{\mathrm{im}}$ Plasmid mini-prep kit (GeneAll). Sequencing of primate DNA samples and alternative transcripts was performed by a commercial sequencing company (Macrogen Inc).

\section{Branch point analysis}

Branch point (BP) analysis was performed using two different programs, svm-BPfinder [36], a widely used program, and BPP, the most recently developed program [34]. These operate on a website and the Linux operating system, respectively. The input sequences ranged from the GT dinucleotide, at the $5^{\prime}$-end of the 4th intron of the BLOC1S2 gene, to the AG dinucleotide, a 3'SS of the target exonized sequence, and genomic PCR sequences of human, chimpanzee, gibbon, crab-eating monkey, rhesus monkey, and African green monkey were used for the analysis. In svm-BPfinder, although many possible BP sequences were predicted based on the distance from the 3 '-SS and scoring information, the most reliable BP sequence that was connected to the target 3'-SS was manually selected according to the instructions of the program. On the other hand, BPP gave only one reliable BP sequence with the programed scoring information.

\section{Combined sequence analysis}

TinT, screening program for nested transposition was introduced in the paper of Churakov et al. [44]. In the program, input file must be RepeatMasker outfile but up-to-date outfile in RepeatMasker website was generated in 2010. Even the outfile used as an input file for the TinT program in the paper of Churakov et al. was generated in 2006. In this study, therefore, we decided to obtain the desired information using our own algorithm on Linux with recently (2016) generated files instead of using TinT program. We calculated the number of TE combination phenomena using Awk, and Python languages in the Linux operating system (Ubuntu 17.04) with the recently annotated data files from the UCSC database. We downloaded repeat-masking files (rmsk.txt.gz) and reference gene files (refGene.txt.gz) for crab-eating monkey, rhesus monkey, and human from $\mathrm{ftp}: / /$ hgdownload.soe.ucsc.edu. We simply parsed the repeat-masking file to count the total number of TEs, SINEs, AluSp elements, and MIR elements and built a Python program algorithm to extract all the combinations in which the TEs are together. Finally, we determined the total number of MIR_AluSp combinations and compared this information with a reference gene file to check how many intragenic MIR_AluSp combinations 
there were. We applied the same algorithm to all three species.

\section{Peptide sequence analysis}

Peptide sequence analysis was performed with ORF finder program and Pfam database. ORF finder program in NCBI website (www.ncbi.nlm.nih.gov/orffinder) is a graphical analysis tool searching for open reading frames (ORFs). To search for possible ORFs and protein sequences of orignal transcript, V1 and V2 respectively, we used each predicted nucleotide sequence as a query sequence. Of the resulting ORFs, we selected one that starts first which could most likely be translated. Afterwards, we do the domain search analysis in the Pfam database website (pfam.xfam.org). In the Pfam website, each protein sequence of the selected ORF is used as a query sequence.

\section{Supplementary information}

Supplementary information accompanies this paper at https://doi.org/10. 1186/s12862-019-1530-0.

Additional file 1: Table S1. Primer list for genomic PCR and RT-PCR Figure S1. Alignment of reported BLOC1S2 m-RNA sequences for crabeating monkey, rhesus monkey and human. Figure S2. Multiple sequence alignment of the integrated MIR and AluSp region in the BLOC1S2 gene. Figure S3. Structural analysis of gDNA PCR products of 10 primates. Figure S4. RT-PCR amplification for validation of the MIR AluSpderived V1 transcript. The 5'- and 3'-ends of MIR_AluSp-derived exon were validated using Validation Primers 1 and 2. A) 531 bp product and $389 \mathrm{bp}$ product of the $\mathrm{V} 1$ transcript were detected in the crab-eating monkey, rhesus monkey, and African green monkey. For the African green monkey, a 506 bp product of a different $5^{\prime}$-end transcript (V2) was detected. B) The $531 \mathrm{bp}$ product and $389 \mathrm{bp}$ product of the $\mathrm{V} 1$ transcript were not detected in the human, chimpanzee, and gibbon samples. Figure S5. RT-PCR amplification for validation of the V2 transcript using Primer 3. A 239 bp product of the V2 transcript was detected in the crabeating monkey, rhesus monkey, and African green monkey and was not detected in human, chimpanzee, and gibbon samples. Figure S6. RT-PCR amplification for expression pattern of V2 transcript (239 bp) in the crabeating monkey (A), rhesus monkey (B), and human (C). The experiments were performed with 35 cycles of $94^{\circ} \mathrm{C}$ for $30 \mathrm{~s}, 59^{\circ} \mathrm{C}$ for $30 \mathrm{~s}$, and $72{ }^{\circ} \mathrm{C}$ for 30 s because 30 cycles of V2 PCR amplification in Fig. 4 doesn't show clear target bands. Figure S7. Multiple alignment of the amino acid sequences of the reference transcript, V1, and V2 transcripts of BLOC1S2. Lysine $(K)$, Leucine $(L)$ in green rectangular part are equivalent to the last part of the 4th exon nucleotide sequences of BLOC1S2 transcript and see Fig. 5 for more details. Table S2. Calculation of MIR_AluSp combination throughout the genome.

\section{Abbreviations}

3'-SS: 3'-splicing site; 5'-SS: 5'-splicing site; AS: Alternative splicing; BP: Branch point; CDS: Coding sequence; HERV: Human endogenous retrovirus; LINE: Long interspersed nuclear elements; NHP: Non-human primate; NWM: New World monkey; OWM: Old World monkey; SINE: Short interspersed nuclear elements; TEs: Transposable elements; TPRT: Targetprimed reverse transcription; TSD: Target site duplication; UTR: Untranslated region

\section{Acknowledgments}

Not applicable.

\section{Authors' contributions}

HMC, SJP, and SHC contributed equally to this work. YHK, JWH, HMC, SJP, and $\mathrm{SHC}$ designed the project. YHK and JWH supervised the progress of the project. HMC, SJP, and SHC performed the experiments. HMC, SJP, SHC and JRL analyzed the data. HMC, SJP, SHC and JRL contributed to drafting of the manuscript. SUK, YBJ, JSK, and SRL contributed reagents and materials and also contributed substantially to the interpretation of the data and the revision of the manuscript. All authors read and approved the final manuscript.

\section{Funding}

This research was supported by a grant from KRIBB Research Initiative Program (KGM4621922 \& KGM5281921). The program funded all related works: study design, specimen collection, molecular experiments, capillary sequencing, data analysis, writing manuscript and publication of the article.

\section{Availability of data and materials}

All data generated or analysed during this study are included in this published article [and its supplementary information files].

\section{Ethics approval}

Human and rhesus monkey RNA samples were purchased at Clontech Laboratories, Inc. Crab-eating monkey, chimpanzee, gibbon and African green monkey samples were provided by the National Primate Research Center (NPRC) of the Korea Research Institute of Bioscience and Biotechnology (KRIBB) in Korea. All procedures for animal sample preparation and the study design were in accordance with the Guidelines of the Institutional Animal Care and Use Committee of the KRIBB (Approval No. KRIBB-AEC-18144).

\section{Consent for publication}

Not applicable.

\section{Competing interests}

The authors declare that they have no competing interests.

\section{Author details}

${ }^{1}$ National Primate Research Center, Korea Research Institute of Bioscience and Biotechnology (KRIBB), Cheongju 28116, Korea. ${ }^{2}$ Department of Functional Genomics, KRIBB School of Bioscience, Korea University of Science \& Technology (UST), Daejeon 34113, Korea. ${ }^{3}$ Primate Resource Center, Korea Research Institute of Bioscience and Biotechnology (KRIBB), Jeongeup 56216, Korea. ${ }^{4}$ Futuristic Animal Resource and Research Center, Korea Research Institute of Bioscience and Biotechnology (KRIBB), Cheongju 28116, Korea.

Received: 19 December 2018 Accepted: 18 October 2019

Published online: 30 October 2019

References

1. Zhou W, He Q, Zhang C, He X, Cui Z, Liu F, Li W. BLOS2 negatively regulates Notch signaling during neural and hematopoietic stem and progenitor cell development. Elife. 2016;5. https://doi.org/10.7554/eLife.18108.

2. Gdynia G, Lehmann-Koch J, Sieber S, Tagscherer KE, Fassl A, Zentgraf H, Matsuzawa S, Reed JC, Roth W. BLOC1S2 interacts with the HIPPI protein and sensitizes NCH89 glioblastoma cells to apoptosis. Apoptosis. 2008;13(3): 437-47

3. Sun J, Nie J, Hao B, Li L, Xing G, Wang Z, Zhou Y, Sun Q, Li G, Zhang L, et al. Ceap/BLOS2 interacts with BRD7 and selectively inhibits its transcriptionsuppressing effect on cellular proliferation-associated genes. Cell Signal. 2008:20(6):1151-8

4. Sammeth M, Foissac S, Guigo R. A general definition and nomenclature for alternative splicing events. PLoS Comput Biol. 2008;4(8):e1000147.

5. Iniguez LP, Hernandez $G$. The evolutionary relationship between alternative splicing and gene duplication. Front Genet. 2017;8:14.

6. Gallego-Paez LM, Bordone MC, Leote AC, Saraiva-Agostinho N, Ascensao-Ferreira M, Barbosa-Morais NL. Alternative splicing: the pledge, the turn, and the prestige : the key role of alternative splicing in human biological systems. Hum Genet. 2017;136(9):1015-42.

7. Ast G. How did alternative splicing evolve? Nat Rev Genet. 2004;5(10): 773-82.

8. Kim E, Goren A, Ast G. Alternative splicing: current perspectives. Bioessays. 2008;30(1):38-47. 
9. Kim YH, Choe SH, Song BS, Park SJ, Kim MJ, Park YH, Yoon SB, Lee Y, Jin YB, Sim BW, et al. Macaca specific exon creation event generates a novel ZKSCAN5 transcript. Gene. 2016;577(2):236-43.

10. Lev-Maor G, Sorek R, Shomron N, Ast G. The birth of an alternatively spliced exon: 3' splice-site selection in Alu exons. Science. 2003;300(5623):1288-91.

11. Park SJ, Kim YH, Lee SR, Choe SH, Kim MJ, Kim SU, Kim JS, Sim BW, Song BS, Jeong KJ, et al. Gain of a new exon by a lineage-specific Alu elementintegration event in the BCS1L gene during primate evolution. Mol Cells. 2015;38(11):950-8.

12. Keren $\mathrm{H}$, Lev-Maor G, Ast G. Alternative splicing and evolution: diversification, exon definition and function. Nat Rev Genet. 2010;11(5):34555.

13. Jjingo $D$, Conley AB, Wang J, Marino-Ramirez L, Lunyak W, Jordan IK. Mammalian-wide interspersed repeat (MIR)-derived enhancers and the regulation of human gene expression. Mob DNA. 2014:5:14

14. Bannert N, Kurth R. Retroelements and the human genome: new perspectives on an old relation. Proc Natl Acad Sci U S A. 2004;101(Suppl 2): 14572-9.

15. Schmitz J, Brosius J. Exonization of transposed elements: a challenge and opportunity for evolution. Biochimie. 2011;93(11):1928-34.

16. Krull M, Petrusma M, Makalowski W, Brosius J, Schmitz J. Functional persistence of exonized mammalian-wide interspersed repeat elements (MIRs). Genome Res. 2007;17(8):1139-45.

17. Gal-Mark N, Schwartz S, Ast G. Alternative splicing of Alu exons--two arms are better than one. Nucleic Acids Res. 2008;36(6):2012-23.

18. Lee JR, Park SJ, Kim YH, Choe SH, Cho HM, Lee SR, Kim SU, Kim JS, Sim BW, Song BS, et al. Alu-derived alternative splicing events specific to Macaca lineages in CTSF gene. Mol Cells. 2017:40(2):100-8

19. Sorek R, Ast G, Graur D. Alu-containing exons are alternatively spliced. Genome Res. 2002:12(7):1060-7.

20. Smit AF, Riggs AD. MIRs are classic, tRNA-derived SINEs that amplified before the mammalian radiation. Nucleic Acids Res. 1995;23(1):98-102.

21. Carnevali D, Conti A, Pellegrini M, Dieci G. Whole-genome expression analysis of mammalian-wide interspersed repeat elements in human cell lines. DNA Res. 2017;24(1):59-69.

22. Huh JW, Kim YH, Park SJ, Kim DS, Lee SR, Kim KM, Jeong KJ, Kim JS, Song BS, Sim BW, et al. Large-scale transcriptome sequencing and gene analyses in the crab-eating macaque (Macaca fascicularis) for biomedical research. BMC Genomics. 2012;13:163.

23. Lee JR, Ryu DS, Park SJ, Choe SH, Cho HM, Lee SR, Kim SU, Kim YH, Huh JW. Successful application of human-based methyl capture sequencing for methylome analysis in non-human primate models. BMC Genomics. 2018: 19(1):267.

24. Kim YH, Park SJ, Choe SH, Lee JR, Cho HM, Kim SU, Kim JS, Sim BW, Song $B S$, Lee $Y$, et al. Identification and characterization of the tyrosinase gene (TYR) and its transcript variants (TYR_1 and TYR_2) in the crab-eating macaque (Macaca fascicularis). Gene. 2017:630:21-7.

25. Cordaux R, Batzer MA. The impact of retrotransposons on human genome evolution. Nat Rev Genet. 2009:10(10):691-703.

26. Lee JR, Kim YH, Park SJ, Choe SH, Cho HM, Lee SR, Kim SU, Kim JS, Sim BW, Song BS, et al. Identification of alternative variants and insertion of the novel polymorphic AluYl17 in TSEN54 gene during primate evolution. Int J Genomics. 2016:2016:1679574.

27. Platt RN 2nd, Vandewege MW, Ray DA. Mammalian transposable elements and their impacts on genome evolution. Chromosom Res. 2018;26(1-2):25-43.

28. Hancks DC, Kazazian HH Jr. Roles for retrotransposon insertions in human disease. Mob DNA. 2016;7:9.

29. Kryatova MS, Steranka JP, Burns KH, Payer LM. Insertion and deletion polymorphisms of the ancient AluS family in the human genome. Mob DNA. 2017:8:6.

30. Dewannieux M, Esnault C, Heidmann T. LINE-mediated retrotransposition of marked Alu sequences. Nat Genet. 2003;35(1):41-8.

31. Ade C, Roy-Engel AM, Deininger PL. Alu elements: an intrinsic source of human genome instability. Curr Opin Virol. 2013;3(6):639-45.

32. Tan J, Ho JX, Zhong Z, Luo S, Chen G, Roca X. Noncanonical registers and base pairs in human 5' splice-site selection. Nucleic Acids Res. 2016:44(8):3908-21.

33. Bitton DA, Rallis C, Jeffares DC, Smith GC, Chen YY, Codlin S, Marguerat S, Bahler J. LaSSO, a strategy for genome-wide mapping of intronic lariats and branch points using RNA-seq. Genome Res. 2014;24(7):1169-79.

34. Zhang Q, Fan X, Wang Y, Sun M, Shao J, Guo D. BPP: a sequence-based algorithm for branch point prediction. Bioinformatics. 2017;33(20):3166-72.
35. Mackereth CD, Madl T, Bonnal S, Simon B, Zanier K, Gasch A, Rybin V, Valcarcel J, Sattler M. Multi-domain conformational selection underlies premRNA splicing regulation by U2AF. Nature. 2011;475(7356):408-11.

36. Corvelo A, Hallegger M, Smith CW, Eyras E. Genome-wide association between branch point properties and alternative splicing. PLoS Comput Biol. 2010;6(11):e1001016.

37. Mercer TR, Clark MB, Andersen SB, Brunck ME, Haerty W, Crawford J, Taft RJ, Nielsen LK, Dinger ME, Mattick JS. Genome-wide discovery of human splicing branchpoints. Genome Res. 2015;25(2):290-303.

38. Signal B, Gloss BS, Dinger ME, Mercer TR. Machine learning annotation of human branchpoints. Bioinformatics. 2018:34(6):920-7.

39. Shao C, Yang B, Wu T, Huang J, Tang P, Zhou Y, Zhou J, Qiu J, Jiang L, Li H, et al. Mechanisms for U2AF to define 3' splice sites and regulate alternative splicing in the human genome. Nat Struct Mol Biol. 2014;21(11):997-1005.

40. Chen L, Weinmeister R, Kralovicova J, Eperon LP, Vorechovsky I, Hudson AJ, Eperon IC. Stoichiometries of U2AF35, U2AF65 and U2 snRNP reveal new early spliceosome assembly pathways. Nucleic Acids Res. 2017:45(4):2051-67.

41. Cho S, Moon H, Loh TJ, Jang HN, Liu Y, Zhou J, Ohn T, Zheng X, Shen H. Splicing inhibition of U2AF65 leads to alternative exon skipping. Proc Natl Acad Sci U S A. 2015;112(32):9926-31.

42. Hilgard P, Huang T, Wolkoff AW, Stockert RJ. Translated Alu sequence determines nuclear localization of a novel catalytic subunit of casein kinase 2. Am J Physiol Cell Physiol. 2002;283(2):C472-83.

43. Tress ML, Abascal F, Valencia A. Alternative splicing may not be the key to proteome complexity. Trends Biochem Sci. 2017;42(2):98-110.

44. Churakov G, Grundmann N, Kuritzin A, Brosius J, Makalowski W, Schmitz J. A novel web-based TinT application and the chronology of the primate Alu retroposon activity. BMC Evol Biol. 2010;10:376.

\section{Publisher's Note}

Springer Nature remains neutral with regard to jurisdictional claims in published maps and institutional affiliations.

Ready to submit your research? Choose BMC and benefit from:

- fast, convenient online submission

- thorough peer review by experienced researchers in your field

- rapid publication on acceptance

- support for research data, including large and complex data types

- gold Open Access which fosters wider collaboration and increased citations

- maximum visibility for your research: over $100 \mathrm{M}$ website views per year

At BMC, research is always in progress.

Learn more biomedcentral.com/submission 